\title{
Visualization, Planning, and Monitoring Software for MRI-Guided Prostate Intervention Robot
}

Emese Balogh ${ }^{1,6}$, Anton Deguet ${ }^{1}$, Robert C. Susil ${ }^{2}$, Axel Krieger ${ }^{3}$, Anand Viswanathan ${ }^{1}$, Cynthia Ménard ${ }^{4}$, Jonathan A. Coleman ${ }^{5}$, and Gabor Fichtinger ${ }^{1,3}$

${ }^{1}$ Engineering Research Center, Johns Hopkins University, Baltimore, MD, USA emese@cs.jhu.edu

2 Dept. of Biomedical Engineering, Johns Hopkins University, Baltimore, MD, USA

3 Dept. of Radiology, Johns Hopkins University, Baltimore, MD, USA

4 Urologic Oncology Branch, NCI, National Institutes of Health, Bethesda, MD

${ }^{5}$ Radiation Oncology Branch, NCI, National Institutes of Health, Bethesda, MD

${ }^{6}$ Dept. of Informatics, University of Szeged, Szeged, Hungary

\begin{abstract}
This paper reports an interactive software interface for visualization, planning, and monitoring of intra-prostatic needle placement procedures performed with a robotic assistant device inside standard cylindrical high-field magnetic resonance imaging (MRI) scanner. We use anatomical visualization and image processing techniques to plan the process and apply active tracking coils to localize the robot in realtime to monitor its motion relative to the anatomy. The interventional system is in Phase-I clinical trials for prostate biopsy and marker seed placement. The system concept, mechanical design, and in-vivo canine studies have been presented earlier 6[10:14]. The software architecture and three-dimensional application software interface discussed in this paper are new additions. This software was tested on pre-recorded patient data.
\end{abstract}

\section{Introduction}

With an estimated annual incidence of 221,000 cases, prostate cancer is the most common cancer in men in the United States and it is responsible for about 29,000 deaths annually 8]. Numerous studies have demonstrated the potential efficacy of needle-based therapy and biopsy procedures in the management of prostate cancer, but contemporary needle delivery techniques still present major limitations. The "Gold Standard" of guiding biopsy and local therapies has been transrectal ultrasound (TRUS) [9]. It is popular due to its real-time nature, low cost, and ease of use, but its limitations are also substantial. Conventional unassisted freehand biopsy techniques have resulted in a low detection rate of 20\%-30\% 12. TRUS has been widely applied in guiding the insertion of metal seeds used for therapeutic irradiation (brachytherapy) and targeting external beam radiation therapy [11, although it fails to visualize the fine details of prostatic tissues. MRI is an attractive choice for image-guidance, because it 


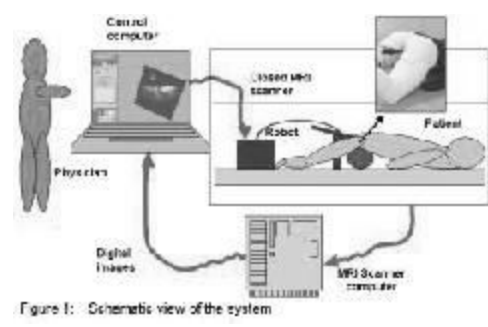

(a)

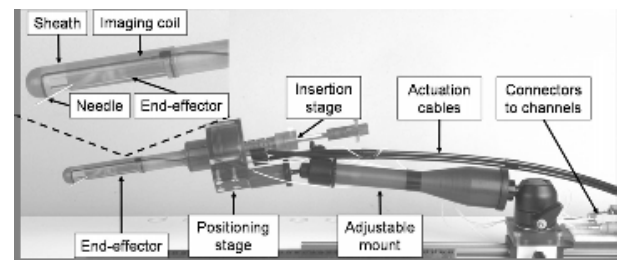

(b)

Fig. 1. System concept (a) 6], Robot assembled prior to treatment (b) [10]

can clearly visualize the prostate and its substructure [1. MRI can also show the distribution and buildup of injected liquid agents, implanted solid capsules, and can monitor the progress of thermal therapies, all in real-time. All facts considered, supplanting TRUS in prostate biopsy and therapy with a bettersuited image guidance modality such as MRI is a logical imperative. However, the strong magnetic field and extremely confined physical space in conventional high-field MRI scanners present formidable physician/patient access challenges. D'Amico et al. manually performed targeted biopsy and brachytherapy inside a 0.5T open MRI scanner [3]4 that tends to have suboptimal image quality. With the use of $1.5 \mathrm{~T}$ conventional MRI, Beyersdorff et al. applied transrectal needle guide [2] and Susil et al. used transperineal template [13. They both used passive fiducial registration and the patient was moved between imaging and the manual needle insertion. In contrast to prior works, we designed a robot to perform the procedure in closed MRI scanner. (We note, however, that for increased safety, in our current protocol we insert the needle outside the magnet.) The robot is operated with real-time tracking and servo control.

\section{System Design}

In our concept (Fig. 1a), the device is secured to the table of the scanner with an adjustable mount. The patient is positioned on the table in prone position with elevated pelvis, the device is introduced to the rectum, and then the patient is moved into the scanner's magnet. The MRI scanner produces signal with the patient and device in the field, at the same time. We determine the spatial relationship between the device and the coordinate system of the MRI scanner using magnetic coils. The images are transferred to a computer that produces a $3 \mathrm{D}$ representation of the device superimposed on the anatomic images. The physician selects the target point for the needle and the computer calculates the kinematic sequence to bring the needle to the selected target position. The computer displays the three motion parameters to the physician who controls the device from outside the magnet. While the actuation of the device is in progress, 
we continue to track the robot's end-effector in real-time. The application computer visualizes the scene with an updated model of the robot, thereby allowing the physician to monitor the motion of the device relative to the anatomy. The robot (Fig. 1b) has three degrees of freedom: (1) a cylindrical end-effector translates in and out the rectum, (2) rotates around the central axis of the translation, and (3) finally the needle is inserted into the prostate. The needle exits on the side of the end-effector and it is inserted into the prostate through the rectum in an oblique angle, along a predefined trajectory, to a predefined depth. There is a rigid stationary sheath around the tubular end-effector. Only the sheath makes contact with the rectum, while the end-effector can freely move inside the sheath, thereby preventing any mechanical distortion to the rectum wall and prostate. The sheath contains an exit window for the needle and imaging coil winding around the window. We employ several guiding channels in the endeffector, providing multiple alternative exit angles. During path planning, the physician can select the optimal angle, depending on the location of the target.

For registration of the robot, we use three active tracking coils attached to the end-effector. The position of each coil in the end-effector is known through calibration. When real-time readings of the coil positions arrive, fast calculation produces the pose of the end-effector in MRI space with an accuracy of 1 $\mathrm{mm}$ in less than $60 \mathrm{~ms}$ time. MRI is a true real-time imaging modality that makes it possible to manipulate the robot's end-effector in a visual servo loop without encoding the joints. The joints are decoupled and non-backdrivable, their motion is linear, smooth, slow, and their relative pose can be estimated with good accuracy. These conditions make it possible to close the visual servo loop through the physician who handles the actuators, without applying encoders or motors on the joints. The physician moves the joints sequentially. The end-effector is registered in real-time and the updated motion parameters tend to zero as the end-effector is approaching the preplanned position. The joints are independently adjusted to finalize the position. This yielded a safe, simple, inexpensive, and versatile system with which an accuracy of $2.0 \mathrm{~mm}$ in-vivo was achieved 1014.

\section{Software Architecture}

The architecture of the software system is shown in Fig. 2a. The complete software is distributed between two computers. One is an SGI Octane (Silicon Graphics, Paolo Alto, CA) that serves as a front-end to a GE $1.5 \mathrm{~T} \mathrm{CV} / \mathrm{I}$ MRI scanner (GE Medical Systems, Waukesha, Wisconsin) and the other is a Windows XP laptop (Dell Products, L.P., Round Rock, TX.) that serves as an Application Computer. The Tracking Server and DICOM Server both run on the scanner's front-end. When new data becomes available on the scanner's front-end computer, the servers automatically push the data to the client side where they are captured in a dedicated storage area. The physician works at the Application Computer running the top-level Slicer-based Application Program that automatically detects the arrival of tracking and image data. The Tracking Server computes the location of the three tracking coils in MRI image space. It is a $\mathrm{C} / \mathrm{C}++$ application that receives MRI signal data from the scanner. To determine the position and orientation of these coils, twelve 1D dodecahedrally spaced 


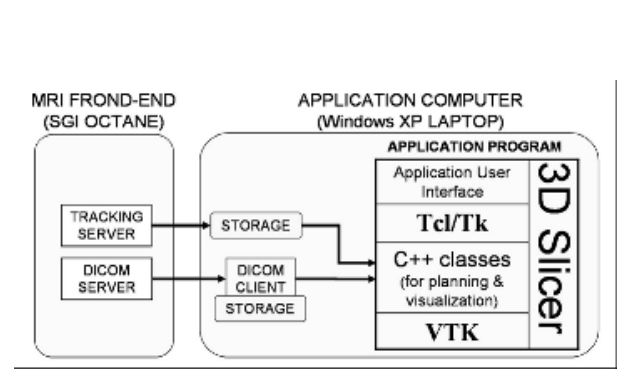

(a)

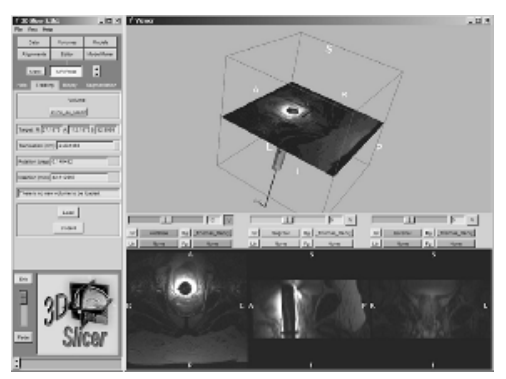

(b)

Fig. 2. Software archtiecture (a) and Slicer-based application program interface (b)

readouts are collected (TE $2.3 \mathrm{msec}$, TR $5.0 \mathrm{msec}$, BW +/-64 KHz, FA 1o, FOV $40 \mathrm{~cm}, 256$ readout points), allowing for coil localization, as described previously in [5]. Microcoil location errors due to gradient nonuniformity are removed using gradient dewarping algorithms obtained from the vendor (GE Medical Systems, Waukesha, Wisconsin). At the end of the pipeline, the Tracking Server produces 9 coordinates and 3 error values for its client through a TCP/IP connection with a frequency of about $2 \mathrm{~Hz}$. When new data arrives to the tracking data storage, the robot's current position and inverse kinematics are recalculated and presented to the physician. The vendor's DICOM Server on the MRI front-end is programmed to send all newly created DICOM series through a local network to the Application Computer specified by an IP address. When new data arrives to the DICOM data storage, the physician can decide whether or not to load the new series into the Slicer viewer.

\section{The Application Program Interface}

The top-level visualization, control, and planning software is implemented within the 3D Slicer package that provides a unified environment for modeling, planning, and monitoring of image guided surgery applications [7]. Slicer integrates into a single environment many aspects of visualization, planning, and monitoring used in image-guided therapies. It provides capabilities for registration, semi-automatic segmentation, generating 3D surface models, and quantitative analysis (measuring distances, angles, surface areas, and volumes) of various medical scans. The 3D Slicer (www.slicer.org) is an open-source software package developed primarily by the Brigham and Women's Hospital. Slicer is extendable due to its modular structure. Each module has its own panel in the Slicer graphical user interface that the developer then flushes out to create his/her own user interface using Tcl/Tk (www.tcl.tk). Modules have access to a wide range of visualization capabilities since Slicer is built on VTK, the Visualization Toolkit (http://www.vtk.org). 
After starting the Slicer-based Application Program, the Menu and the Viewer windows appear (Fig. 2b). The Menu window provides menus for accessing the features of Slicer, while the Viewer window displays volumes, image slices, and models. The upper part of the Viewer window is the $3 \mathrm{D}$ viewer, while the lower part displays 2D slices. The $2 \mathrm{D}$ images can be either original or reformatted images along orthogonal cutting planes across the MRI volume. The $2 \mathrm{D}$ images can also be texture-mapped onto a plane and rendered in the $3 \mathrm{D}$ view in their correct location; that is, where the reformatted voxels exist in the volume. Each 2D image and the 3D view may be zoomed independently. A graphical representation of the robot's end-effector (Fig. 3a) appears superimposed in the 3D view. At any time during planning and intervention, the physician can visualize the robot relative to the $3 \mathrm{D}$ volume of the patient's image. We enhance these visual effects by the built-in visualization tools and routines of VTK and Slicer packages, such as changing transparency of objects and so forth. The robot's position is also projected onto the slices in the $2 \mathrm{D}$ windows. The target and intersection of the needle's trajectory with the slice are both presented, thereby giving the physician yet another visual clue whether the needle is being correctly oriented toward the target.

When the target is selected by the physician on a 2D axial slice, the program immediately computes the inverse kinematics of the robot to reach the selected target. The motion parameters are calculated for all alternative needle exit angles and program generates a warning message if the target cannot be reached by one of the needles due to exceeding the allotted range of motion or major anatomical constraints. The physician then selects the appropriate needle channel and the rotation, translation, and insertion are displayed, so that the motion of the robot can commence. The computer can also simulate the sequence of motions by moving a 3D model of the device, to allow the physician to verify that the calculated sequence of motions would indeed take the needle from its current position to the pre-selected target position.

During the robot movements, tracking coil positions are refreshed at a frequency of about $2 \mathrm{~Hz}$. The inverse kinematics is also recalculated and presented to the physician. The robot's position is updated in the $2 \mathrm{D}$ and $3 \mathrm{D}$ viewers. As the physician moves the device, the end-effector's model appears to be moving toward the target in the $3 \mathrm{D}$ view (Fig $3 \mathrm{~b}$ ). When all motion parameters reach zero and the needle points toward the target (Fig 3c), the physician may start inserting the needle to the predetermined depth. In the current embodiment, the needle insertion is not encoded, but the needle can be observed in real-time MRI images. A full volume is also acquired after the needle is inserted, but before the biopsy sample is cut or the marker seed is released. The visualization, motion planning, and monitoring workflow and software were designed to uniformly support a plurality of needle-based interventions. This approach even allows the physician to perform combined interventions. For example, the physician can use the system to place marker seeds into the prostate, and in the same session can also collect biopsy samples if suspicious nodes are detected in the MRI images since the last imaging or biopsy session. (It is never too late to update the staging of the disease, which is the decisive factor in selecting the right course of treatment.) 


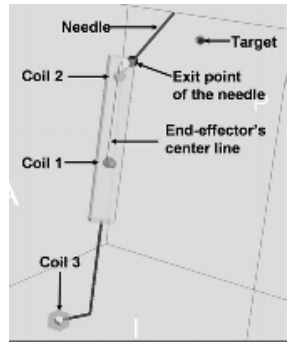

(a)

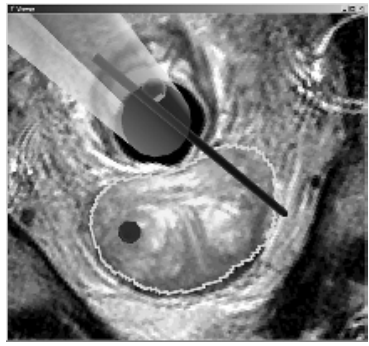

(b)

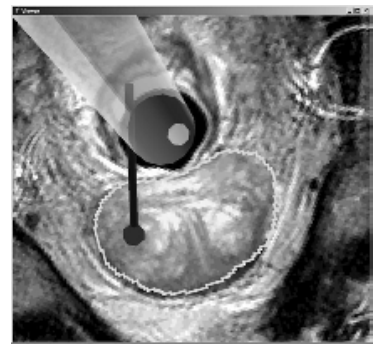

(c)

Fig. 3. Model of the end-effector (a). Robot tracking in the 3D viewer. The model of the end-effector and trajectory of needle are superimposed on the pre-segmented target image. When the needle's trajectory intersects with the target (c) the robot can stop.

\section{Validation}

The accuracy of tracking was verified with the use of a precisely machined mechanical frame that allowed for controlled translations and rotations. This allowed us to move the tracking coils through predefined trajectories and verify whether the Tracking Server correctly rectifies these trajectories. The three tracking coils were fixed to form a triangle that we tracked through a series of poses in the MRI scanner. We calculated the trajectory of the centroid and plane normal vector of the triangle and compared those to the a priori known trajectory. The differences for the centroid and plane normal were $0.41 \mathrm{~mm}$ and 1.1 degree, respectively. The fully assembled robot was placed in the MRI scanner and ascertained that incremental motions of the rotation and translation joints correspond to the incremental rotations and translations rectified from the tracking coils. On the client side, we measured an update rate of $2 \mathrm{~Hz}$, which sufficed the polling rate in the top-level application software and was also adequate for the speed of the robotic assistance device. The functionality, speed, robustness, and correctness of the DICOM client/server interface were tested on multiple series of phantom and patient data. Each new DICOM series appeared on the Application Computer with an acceptable delay, considering the amount of data transferred. The contents of the DICOM series were compared between the server and client sides. No loss of information occurred during the transfer and interpretation of the images. The Tracking and DICOM Server-Client units have been integrated under a simplified 2D application interface and deployed first in canine experiments [14] and in Phase-I human trials for prostate biopsy and marker seed placement.

The Slicer-based Application Program Interface has been tested in a simulated environment using the Tracking and DICOM Servers with pre-recorded data from human and phantom cases. The goal was to demonstrate that the Slicer-based Application Program Interface performs as accurately and reliably as the previously clinically commissioned 2D application interface. First we as- 
certained that the 3D program provides at least the same numerical accuracy, visual realism, and clinical functionality as the simpler $2 \mathrm{D}$ version, and then we verified that the new 3D functions produce correct results. In particular, we conducted the following series of tests. (1) We verified that all 2D images and pixels coordinates presented in the Slicer-based interface corresponded to the images and pixel values on the MRI scanners console, which we considered ground truth. (2) By running the inverse kinematics of the robot through the same series of targets as in the pre-recorded cases, we verified that the calculation of robot motion parameters were correct. (3) By cutting through the MRI volume with texture mapped planes in canonical and oblique angles, we verified that all reconstructed surface models of the anatomy (prostate, urethra, nerve bundles, bladder, and rectum) corresponded to the MRI volume. (4) By superimposing the bright spots produced by the Gadolinium fiducials built in the robot and the graphical model of the robot, we verified that the tracking server provided spatially accurate information and that they appeared correctly in the $3 \mathrm{D}$ viewer.

\section{Conclusions}

The $3 \mathrm{D}$ visualization, planning, and monitoring system based on the $3 \mathrm{D}$ Slicer has been found to be a functionally viable solution. The current embodiment of the application interface software appears to be applicable in clinical use. Currently, we are in the process of migrating the ongoing biopsy and marker seed placement clinical trials from a $1.5 \mathrm{~T}$ GE scanner to a $3 \mathrm{~T}$ machine of an other vendor, which necessitates further customization in the software. We have found that the application of active tracking coils for robot guidance is a most accurate and versatile solution, but it also requires significant interaction and exchange of proprietary information with the scanner's vendor. The 3D Slicer system, although it is highly customizable, was not created with the primary intention of clinical use and thus some aspects of its ergonomics still needs further tuning. For the clinicians, handling reconstructed $3 \mathrm{D}$ views is a departure from conventional $2 \mathrm{D}$ slices, requiring off-line practice. We have determined in the ongoing clinical trials that organ deformation and motion still remains a significant factor. These negative effects could be reduced by a model predicting the deformation and dislocation of the target and thereby allowing for compensation in the planning phase. This, however, is a long-term objective that also involves rapid anatomical segmentation and signal inhomogeneity correction.

Acknowledgments. We are grateful to L.L. Whitcomb and E. Atalar for their contribution. Support was provided by NIH R01 EB002963, HL57483, HL61672, NSF 9731478, US Army PC 10029.

\section{References}

1. S. Adusumilli and E.S. Pretorius. Magnetic resonance imaging of prostate cancer. Semin Urol Oncol, 20(3):192-210, 2002. 
2. D. Beyersdorff, A. Winkel, P. Bretschneider, B.K. Hamm, S.A. Loening, and M. Taupitz. Initial results of MRI-guided prostate biopsy using a biopsy device in a closed MR imager at 1.5T. In The 88th Scientific Assembly and Annual Meeting of the Radiological Society of North America, Chicago, page 629, 2002.

3. A.V. D'Amico, C.M. Tempany CM, R. Cormack, N. Hata, M. Jinzaki, K. Tuncali, M. Weinstein, and J.P. Richie. Transperineal magnetic resonance image guided prostate biopsy. J. Urol, 164(2):385-387, 2000.

4. A.V. D'Amico, R. Cormack, C.M. Tempany, S. Kumar, G. Topulos, H.M. Kooy, and C.N. Coleman. Realtime magnetic resonance image-guided interstitial brachytherapy in the treatment of select patients with clinically localized prostate cancer. Int J Radiat Oncol Biol Phys, 42(3):507-515, 1998.

5. J.A. Derbyshire, G.A. Wright, R.M. Henkelman, and R.S. Hinks. Dynamic scanplane tracking using MR position monitoring. J Magn Reson Imaging, 8(4):924932, 1998.

6. G. Fichtinger, A. Krieger, R.C. Susil, A. Tanacs, L.L. Whitcomb, and E. Atalar. Transrectal prostate biopsy inside closed MRI scanner with remote actuation, under real-time image guidance. In Fifth International Conference on Medical Image Computing and Computer-Assisted Intervention, volume 2488 of Lecture Notes in Computer Science, pages 91-98. Springer Verlag, 2002.

7. D.T. Gering, A. Nabavi, R. Kikinis, N. Hata, L.J. O'Donnell, W.E. Grimson, F.A. Jolesz, P.M. Black, and W.M. Wells 3rd. An integrated visulalization system for surgical planning and guidance using image fusion and an open MR. J Magn Reson Imaging, 13(6):967-975, 2001.

8. A. Jemal, T. Murray, A. Samuels, A. Ghafoor, E. Ward, and M. Thun. Cancer statistics, 2003. CA Cancer J Clin, 53:5-26, 2003.

9. J.C. Presti Jr. Prostate cancer: assessment of risk using digital rectal examination, tumor grade, prostate-specific antigen, and system biopsy. Radiol Clin North Am, 38(1):49-58, 2000.

10. A. Krieger, R.C. Susil, G. Fichtinger, E. Atalar, and L.L. Whitcomb. Design of a novel MRI compatible manipulator for image guided prostate intervention. IEEE 2004 International Conference on Robotics and Automation, (accepted), 2004.

11. A.J. Nederveen, J.J. Lagendijk, and P. Hofman. Feasibility of automatic marker detection with an a-si flat-panel imager. Phys Med Biol, 46(4):1219-1230, 2001.

12. K.A. Roehl, J.A. Antenor, and W.J. Catalona. Serial biopsy results in prostate cancer screening study. J. Urol, 167:1156-1161, 2002.

13. R.C. Susil, K. Camphausen, P. Choyke, E. Atalar, C. Coleman, and C. Menard. A system for transperineal prostate biopsy and HDR brachytherapy under 1.5T MRI guidance. techniques and clinical experience. In The 89th Scientific Assembly and Annual Meeting of the Radiological Society of North America (RSNA), Chicago, page 644, 2003.

14. R.C. Susil, A. Krieger, J.A. Derbyshire, A. Tanacs, L.L. Whitcomb, E.R. McVeigh, G. Fichtinger, and E. Atalar. A system for MRI guided diagnostic and therapeutic prostate interventions. Journal of Radiology, 228:886-894, 2003. 